\title{
On the approximation of the concentration parameter for von Mises distribution
}

\author{
Nor Hafizah Moslim ${ }^{\mathrm{a}, \mathrm{b}}$, Yong Zulina Zubairi ${ }^{\text {b, }}$, Abdul Ghapor Hussin ${ }^{\mathrm{c}}$, Siti Fatimah Hassan ${ }^{\mathrm{b}}$, \\ Rossita Mohamad Yunus ${ }^{\text {d }}$ \\ a Faculty of Industrial Sciences \& Technology, Universiti Malaysia Pahang, 26300 Gambang, Pahang \\ ${ }^{b}$ Centre for Foundation Studies in Science, University of Malaya, 50603 Kuala Lumpur \\ c Faculty of Defence Sciences and Technology, National Defence University of Malaysia, Kem Sungai Besi, 57000 Kuala Lumpur \\ ${ }^{d}$ Institute of Mathematical Sciences, Faculty of Science, University of Malaya, 50603 Kuala Lumpur \\ * Corresponding author: yzulina@um.edu.my
}

\section{Article history}

Received 13 October 2017

Accepted 8 November 2017

\begin{abstract}
The von Mises distribution is the 'natural' analogue on the circle of the Normal distribution on the real line and is widely used to describe circular variables. The distribution has two parameters, namely mean direction, $\mu$ and concentration parameter, $\mathrm{k}$. Solutions to the parameters, however, cannot be derived in the closed form. Noting the relationship of the $\mathrm{k}$ to the size of sample, we examine the asymptotic normal behavior of the parameter. The simulation study is carried out and KolmogorovSmirnov test is used to test the goodness of fit for three level of significance values. The study suggests that as sample size and concentration parameter increase, the percentage of samples follow the normality assumption increase.
\end{abstract}

Keywords: circular variable, concentration parameter, Monte Carlo, von Mises

(c) 2017 Penerbit UTM Press. All rights reserved

\section{INTRODUCTION}

Statistical data can be classified according to their distributional topologies. A linear data set can be represented on a straight line and for circular data, they can be represented by the circumference of a circle. The data is commonly measured in the range of $\left(0^{\circ}, 360^{\circ}\right]$ degree or $(0,2 \pi]$ radian. It is worthwhile to note that statistical theories for straight line and circle are very different from one to another because the circle is a closed curve but line is not. The application of directional statistics can be found in the area of meteorology such as wind direction. Circular data can be found in many fields (Mardia, 1972; Mardia et al., 2000; Amos, 1974).

A circular random variable from a von Mises or Circular Normal distribution has a density function of

$$
f(\theta)=\frac{1}{2 \pi I_{0}(\kappa)} e^{\kappa \cos (\theta-\mu)}, 0 \leq \theta \leq 2 \pi
$$

where $0 \leq \mu \leq 2 \pi$ and $\kappa \geq 0$ are the parameters. $I_{0}(\kappa)$ is the modified Bessel function of order zero and can be defined as

$$
I_{0}(\kappa)=\frac{1}{2 \pi} \int_{0}^{2 \pi} e^{\kappa \cos (\theta-\mu)} d \theta
$$

This distribution is the 'natural' analogue on the circle of the normal distribution on the real line and has few similar characteristics with the normal distribution (Dobson, 1978).
It has been proved that the von Mises distribution can be approximated to the standard normal distribution for sufficiently large $\kappa$ (Jammalamadaka et al., 2001; Fisher, 1993). As $\kappa \rightarrow \infty$,

$$
\beta=\sqrt{\kappa}(\theta-\mu) \stackrel{d}{\Rightarrow} N(0,1)
$$

Let $\beta=\sqrt{\kappa}(\theta-\mu)$. For large $\kappa, \theta-\mu=\frac{\beta}{\sqrt{\kappa}}$ is small and from the Taylor series expansion $\cos \theta=1-\frac{\theta^{2}}{2}$, it gives

$$
\begin{aligned}
\cos (\theta-\mu) & =\cos \left(\frac{\beta}{\sqrt{\kappa}}\right) \\
& =1-\frac{\beta^{2}}{2 \kappa}
\end{aligned}
$$

Using the change of variable formula,

$$
\begin{aligned}
g(\beta) & =\left|\frac{\partial \theta}{\partial \beta}\right| f\left(g^{-1}(\beta)\right) \\
& =\frac{1}{\sqrt{\kappa}} \frac{e^{\kappa \cos \left(\frac{\beta}{\sqrt{\kappa}}\right)}}{2 \pi I_{0}(\kappa)}
\end{aligned}
$$


The Bessel functions, $I_{0}(\kappa)$ can be approximated to

$$
I_{0}(\kappa) \sim \frac{e^{\kappa}}{\sqrt{2 \pi \kappa}} \text { for large } \kappa
$$

Thus, by substituting the Eq. (1) and (3) into Eq. (2),

$$
\begin{aligned}
g(\beta) & =\frac{1}{\sqrt{\kappa}} \frac{e^{k \cos \left(\frac{\beta}{\sqrt{\kappa}}\right)}}{2 \pi \frac{e^{\kappa}}{\sqrt{2 \pi \kappa}}} \\
& =\frac{e^{\kappa\left(1-\frac{\beta^{2}}{2 \kappa}\right)}}{e^{\kappa} \sqrt{2 \pi}} \\
& =\frac{1}{\sqrt{2 \pi}} e^{\left(-\frac{\beta^{2}}{2}\right)} \sim N(0,1)
\end{aligned}
$$

From the substitution above, the distribution $g(\beta)$ is approximate to the standard normal distribution. The simulation study should be carried out to test the approximation (Abuzaid et al., 2012).

\section{SIMULATION STUDY}

Simulation studies were conducted for fourteen different sample sizes, $n=5,10,20,30,40,50,60,70,80,90,100,120,150$ and 200 respectively with various values of concentration parameter, $\kappa=1,2$, $3,4,5,6,7,8,9,10,12,15,17,20,25,30,35$ and 45 for three different levels of significance, $\alpha=0.01,0.05,0.10$. The mean direction, $\mu$ is fixed at $\frac{\pi}{4}$. The 3000 simulation of sample data were generated from von Mises distribution. For each generated sample, get the value of $\beta=\sqrt{\kappa}(\theta-\mu)$. Use the Kolmogorov-Smirnov test to test the goodness-of-fit for each value of $\beta$ under the three levels of significance.

Table 1 shows the percentage of samples that follow Standard Normal Distribution, $N(0,1)$. The results were given under the level of significance, $\alpha=0.01,0.05,0.10$ for the first, second and third rows, respectively.

At $10 \%$ of significance level, for any fixed $n$, the percentage of samples that follow the standard normal distribution increase as the concentration parameter, $\kappa$ increase. This is there for $5 \%$ and $1 \%$, respectively.
Also, at any significance level, for any fixed $\kappa<10$, increase in $n$ results is decrease in the percentage of samples that follow the standard normal distribution. But for $\kappa \geq 10$, as $n$ increase the percentage remains constant approximate at $90 \%, 95 \%$ and $99 \%$ for the significance level of $10 \%, 5 \%$ and $1 \%$, respectively.

Of the three levels of significance, $\alpha=0.01$ shows most percentage of samples follow standard normal distribution for any value of concentration parameter, $\kappa$ and sample size, $n$. Small sample size $(n \leq 30)$ shows more than $96 \%$ of samples follow standard normal distribution for $\kappa \geq 5$. Concentration parameter $\kappa$ shows more than $96 \%$ of samples follow standard normal distribution for large sample size.

More than $98 \%$ of generated samples follow standard normal distribution for any sample size when the concentration parameter, $\kappa \geq 9$.

\section{CONCLUSION}

In summary, the generated samples of von Mises distribution for concentration parameter, $\kappa \geq 5$ is approximated to the standard normal distribution. As the sample size, $n$ and concentration parameter, $\kappa$ increase, the percentages of samples follow standard normal distribution increase regardless of the levels of significance values.

\section{ACKNOWLEDGEMENT}

We would like to thank Ministry of High Education (MOHE), STEM Grant with vote no. A. J091002.5600.07397 and research grant RG369-15AFR from University of Malaya for the financial assistance.

\section{REFERENCES}

Jammalamadaka, S. R. and Sengupta, A. 2001. Topics in Circular Statistics. London: World Scientific.

Mardia, K. V. 1972. Statistics of Directional Data. London: Academic Press.

Mardia, K. V. and Jupp, P. E. 2000. Directional Statistics. England: John Wiley \& Sons Ltd.

Abuzaid, A. H., Hussin, A. G., Rambli, A. and Mohamed, I. 2012. Statistics for a new test of discordance in circular data. Communications in Statistics - Simulation and Computation. 41, 1882-1890.

Amos D. E. 1974. Computation of modified bessel functions and their ratios. Mathematics of Computation. 28(125), 239-251.

Dobson A. J. 1978. Simple approximations for the Von Mises concentration statistic. Journal of the Royal Statistical Society, Series C (Applied Statistics). 27(3), 345-347.

Fisher N. 1. 1993. Statistical Analysis of Circular Data. Canberra: Cambridge University Press. 
Moslim et al. / Malaysian Journal of Fundamental and Applied Sciences Special Issue on Some Advances in Industrial and Applied Mathematics (2017) 390-393

Table 1 The percentage of samples in approximating standard normal distribution.

\begin{tabular}{|c|c|c|c|c|c|c|c|c|c|c|c|c|c|c|}
\hline & \\
\hline & 5 & 10 & 20 & 30 & 40 & 50 & 60 & 70 & 80 & 90 & 100 & 120 & 150 & 200 \\
\hline \multirow{3}{*}{1} & 3.70 & 28.47 & 5.20 & 0.00 & 0.00 & 0.00 & 0.00 & 0.00 & 0.00 & 0.00 & 0.00 & .00 & 0.00 & 0.00 \\
\hline & 64.70 & 43.00 & 11.90 & 1.37 & 0.00 & 0.00 & 0.00 & 0.00 & 0.00 & 0.00 & 0.00 & 0.00 & 0.00 & 0.00 \\
\hline & 85.00 & 65.13 & 32.30 & 10.70 & 2.77 & 0.47 & 0.00 & 0.00 & 0.00 & 0.00 & 0.00 & 0.00 & 0.00 & 0.00 \\
\hline \multirow{3}{*}{2} & 73.30 & 59.50 & 35.23 & 20.33 & 9.97 & 4.70 & 2.30 & 0.67 & 0.10 & 0.00 & 0.00 & 0.00 & 0.00 & 0.00 \\
\hline & 81.40 & 73.23 & 48.50 & 32.93 & 18.93 & 10.90 & 6.50 & 2.90 & 1.17 & 0.40 & 0.03 & 0.00 & 0.00 & 0.00 \\
\hline & 94.43 & 88.40 & 73.43 & 58.97 & 45.57 & 30.87 & 23.70 & 13.43 & 9.57 & 5.10 & 3.07 & 0.23 & 0.00 & 0.00 \\
\hline \multirow{3}{*}{3} & 82.37 & 75.63 & 63.83 & 53.20 & 43.63 & 35.47 & 29.70 & 21.03 & 15.33 & 12.87 & 9.47 & 4.30 & 1.47 & 0.00 \\
\hline & 89.40 & 84.77 & 76.37 & 65.83 & 57.83 & 49.93 & 43.57 & 34.03 & 27.00 & 22.90 & 18.83 & 10.23 & 4.03 & 0.57 \\
\hline & 96.93 & 95.20 & 91.57 & 86.07 & 80.13 & 74.77 & 70.37 & 63.90 & 54.27 & 48.13 & 44.73 & 33.33 & 18.00 & 6.20 \\
\hline \multirow{3}{*}{4} & 85.23 & 84.00 & 80.27 & 72.57 & 67.53 & 63.87 & 59.57 & 53.57 & 48.93 & 45.23 & 39.67 & 29.77 & 23.63 & 11.10 \\
\hline & 91.80 & 91.43 & 88.03 & 83.30 & 78.70 & 76.23 & 72.93 & 67.90 & 64.40 & 60.30 & 54.53 & 44.27 & 35.53 & 21.07 \\
\hline & 98.10 & 97.50 & 96.83 & 94.63 & 92.00 & 91.37 & 89.23 & 86.37 & 85.27 & 82.83 & 79.00 & 73.77 & 65.83 & 50.13 \\
\hline \multirow{3}{*}{5} & 88.13 & 88.27 & 84.77 & 82.57 & 78.03 & 76.87 & 75.57 & 73.03 & 71.90 & 67.13 & 66.23 & 62.00 & 53.17 & 41.20 \\
\hline & 94.03 & 94.13 & 91.77 & 89.57 & 86.60 & 86.60 & 85.40 & 82.80 & 81.60 & 79.03 & 78.40 & 75.40 & 66.70 & 55.90 \\
\hline & 98.87 & 98.77 & 98.20 & 97.27 & 96.53 & 95.93 & 95.43 & 94.80 & 94.63 & 93.30 & 93.17 & 90.60 & 86.53 & 80.53 \\
\hline \multirow{3}{*}{6} & 89.73 & 89.30 & 86.80 & 86.80 & 84.67 & 83.87 & 83.90 & 81.53 & 81.20 & 79.43 & 78.90 & 76.60 & 73.00 & 64.80 \\
\hline & 94.50 & 94.77 & 93.23 & 92.67 & 92.23 & 91.07 & 91.17 & 89.67 & 89.10 & 88.20 & 87.30 & 85.63 & 83.47 & 76.73 \\
\hline & 98.87 & 98.83 & 98.60 & 98.17 & 98.03 & 97.80 & 97.30 & 97.57 & 97.10 & 96.87 & 96.17 & 95.80 & 95.03 & 92.10 \\
\hline \multirow{3}{*}{7} & 89.33 & 88.43 & 88.53 & 87.60 & 87.33 & 86.67 & 86.87 & 86.77 & 84.73 & 85.37 & 85.40 & 82.60 & 81.73 & 77.97 \\
\hline & 94.20 & 94.13 & 94.63 & 93.67 & 93.07 & 92.40 & 92.87 & 92.37 & 91.60 & 91.77 & 92.57 & 90.20 & 90.37 & 86.67 \\
\hline & 99.13 & 98.57 & 99.17 & 98.33 & 98.47 & 98.30 & 97.87 & 98.27 & 97.93 & 98.13 & 98.20 & 98.20 & 97.60 & 96.47 \\
\hline \multirow{3}{*}{8} & 90.10 & 88.93 & 88.03 & 88.57 & 88.77 & 88.90 & 88.37 & 88.53 & 87.83 & 87.77 & 87.87 & 87.53 & 86.27 & 83.77 \\
\hline & 94.93 & 94.23 & 93.77 & 94.10 & 94.47 & 94.20 & 94.03 & 94.10 & 93.00 & 93.27 & 93.70 & 93.83 & 92.17 & 90.63 \\
\hline & 99.17 & 98.60 & 98.63 & 98.80 & 98.63 & 98.50 & 98.73 & 98.67 & 98.63 & 98.70 & 98.93 & 98.10 & 97.93 & 97.63 \\
\hline \multirow{3}{*}{9} & 89.87 & 89.17 & 89.70 & 90.03 & 89.87 & 88.93 & 89.70 & 89.67 & 89.80 & 90.33 & 88.53 & 90.70 & 89.43 & 86.50 \\
\hline & 95.00 & 94.33 & 94.60 & 95.27 & 94.47 & 93.97 & 95.17 & 94.57 & 94.57 & 95.33 & 94.67 & 94.93 & 94.07 & 92.43 \\
\hline & 99.27 & 98.70 & 98.83 & 99.20 & 98.90 & 99.00 & 99.10 & 99.00 & 99.03 & 99.13 & 98.90 & 99.00 & 98.70 & 98.37 \\
\hline \multirow{3}{*}{10} & 89.90 & 90.00 & 89.23 & 90.20 & 89.43 & 89.37 & 91.13 & 90.83 & 90.70 & 89.80 & 90.90 & 89.50 & 88.50 & 89.33 \\
\hline & 95.60 & 95.13 & 94.77 & 95.33 & 94.93 & 95.30 & 95.63 & 95.73 & 95.43 & 94.93 & 95.37 & 94.77 & 94.07 & 94.30 \\
\hline & 99.07 & 99.17 & 98.97 & 98.83 & 99.10 & 98.83 & 99.00 & 99.20 & 99.07 & 98.97 & 99.27 & 98.60 & 98.70 & 98.77 \\
\hline \multirow{3}{*}{12} & 88.77 & 89.33 & 89.63 & 89.17 & 89.47 & 89.47 & 89.63 & 90.33 & 89.97 & 90.63 & 89.83 & 90.43 & 91.30 & 90.27 \\
\hline & 94.57 & 94.67 & 94.30 & 94.33 & 94.33 & 94.77 & 94.70 & 95.47 & 95.40 & 95.23 & 94.80 & 95.07 & 96.03 & 95.07 \\
\hline & 99.10 & 98.97 & 98.80 & 98.83 & 98.97 & 99.17 & 99.13 & 99.30 & 99.23 & 99.23 & 98. & 99.07 & 99.20 & 99.07 \\
\hline \multirow{3}{*}{15} & 90.43 & 89.30 & 89.47 & 89.57 & 90.10 & 89.67 & 90.67 & 91.03 & 90.90 & 90.67 & 91.00 & 90.30 & 90.90 & 90.73 \\
\hline & 94.97 & 94.57 & 95.03 & 94.97 & 94.83 & 95.10 & 95.23 & 95.73 & 95.60 & 95.40 & 95.77 & 95.60 & 95.33 & 95.80 \\
\hline & 99.07 & 98.93 & 98.90 & 98.80 & 99.13 & 98.97 & 99.17 & 99.27 & 99.33 & 99.03 & 99.00 & 99.40 & 99.17 & 99.17 \\
\hline \multirow{3}{*}{17} & 90.23 & 89.63 & 89.50 & 89.47 & 89.67 & 89.87 & 90.80 & 91.40 & 91.20 & 90.20 & 90.47 & 90.00 & 90.83 & 90.97 \\
\hline & 95.43 & 94.93 & 95.10 & 94.70 & 94.83 & 95.30 & 95.33 & 95.43 & 95.63 & 95.07 & 95.67 & 95.53 & 95.53 & 95.30 \\
\hline & 99.03 & 98.90 & 98.90 & 98.67 & 99.13 & 98.97 & 99.20 & 99.43 & 99.20 & 99.23 & 99.10 & 99.23 & 99.20 & 99.30 \\
\hline \multirow{3}{*}{20} & 90.10 & 89.33 & 89.60 & 90.43 & 90.10 & 89.83 & 90.47 & 90.27 & 91.77 & 90.97 & 91.77 & 91.10 & 91.23 & 91.17 \\
\hline & 95.23 & 94.43 & 94.83 & 94.67 & 94.70 & 95.00 & 95.10 & 95.00 & 95.67 & 95.47 & 95.77 & 95.60 & 95.63 & 95.70 \\
\hline & 99.07 & 99.07 & 98.97 & 98.73 & 98.97 & 98.70 & 99.20 & 98.83 & 99.07 & 99.10 & 99.03 & 99.00 & 98.93 & 99.37 \\
\hline & 89.97 & 88.80 & 90.07 & 89.60 & 89.17 & 89.70 & 90.43 & 90.47 & 90.83 & 90.37 & 90.53 & 90.87 & 90.57 & 90.37 \\
\hline & 94.93 & 94.67 & 94.87 & 94.50 & 94.03 & 94.50 & 95.27 & 95.07 & 95.73 & 95.90 & 95.43 & 95.07 & 95.43 & 95.27 \\
\hline
\end{tabular}


Moslim et al. / Malaysian Journal of Fundamental and Applied Sciences

Special Issue on Some Advances in Industrial and Applied Mathematics (2017) 390-393

\begin{tabular}{|c|c|c|c|c|c|c|c|c|c|c|c|c|c|c|}
\hline & 98.93 & 98.97 & 99.10 & 98.87 & 98.73 & 99.03 & 99.07 & 98.80 & 99.23 & 99.03 & 98.97 & 98.87 & 99.07 & 99.00 \\
\hline \multirow{3}{*}{30} & 90.53 & 90.20 & 89.90 & 90.43 & 88.83 & 90.30 & 90.03 & 91.80 & 90.67 & 90.70 & 91.23 & 91.10 & 91.50 & 90.13 \\
\hline & 95.47 & 94.43 & 95.27 & 95.47 & 93.97 & 95.23 & 94.87 & 96.13 & 95.77 & 95.33 & 96.03 & 95.57 & 95.93 & 94.70 \\
\hline & 99.17 & 98.83 & 98.87 & 98.90 & 98.70 & 99.03 & 99.17 & 99.33 & 99.17 & 99.10 & 99.27 & 99.10 & 99.47 & 98.80 \\
\hline \multirow{3}{*}{35} & 89.87 & 90.03 & 89.97 & 90.50 & 88.73 & 89.80 & 91.13 & 90.97 & 90.77 & 91.13 & 91.67 & 91.47 & 90.30 & 89.83 \\
\hline & 94.73 & 94.53 & 94.93 & 95.47 & 94.70 & 94.67 & 95.47 & 95.73 & 95.47 & 95.50 & 95.83 & 95.63 & 94.43 & 94.83 \\
\hline & 98.93 & 98.73 & 98.87 & 98.83 & 98.87 & 98.70 & 99.13 & 99.43 & 99.17 & 99.20 & 99.30 & 98.90 & 99.07 & 99.23 \\
\hline \multirow{3}{*}{45} & 89.73 & 89.20 & 90.20 & 89.63 & 90.23 & 90.57 & 91.13 & 90.50 & 91.23 & 90.53 & 90.67 & 90.40 & 90.63 & 90.63 \\
\hline & 95.60 & 94.70 & 94.93 & 94.47 & 94.97 & 94.73 & 95.63 & 95.40 & 95.53 & 95.17 & 95.37 & 95.43 & 95.57 & 95.67 \\
\hline & 98.70 & 99.13 & 98.93 & 99.10 & 99.03 & 98.97 & 99.13 & 99.07 & 98.90 & 99.23 & 99.10 & 99.13 & 99.27 & 99.23 \\
\hline
\end{tabular}

